\title{
Response to ART among adult PLHIV: a prospective study at HIV clinic Colombo
}

\author{
Rajapaksa L .I. ${ }^{1}$, Herath $\mathrm{N}^{2}$., Aryaratne K. A. M. ${ }^{1}$, Weerasinghe G. ${ }^{1}$
}

\begin{abstract}
Introduction: Sri Lanka has initiated ART programme in 2004. By the end of 2014 a total of 825 patients were receiving care services with 481 at the HIV clinic, Colombo. Based on WHO consolidated guidelines on ART published in 2013 the ART guideline was updated in 2014. It was decided to determine the response to ART among PLHIV managed according to the new guideline. The objective of the study was to determine the response to ART among adult PLHIV attending HIV clinic, NSACP.
\end{abstract}

Methods: A clinic based prospective study was carried out at the main STD/HIV clinic in Colombo to assess the clinical and laboratory response to ART. All adult PLHIV who were started on ART from 1st November 2014 to 31st October 2015 were included in the study and were followed up till end October 2016. Data was collected using interviewer administered questionnaire by trained medical officers. Laboratory data and other relevant information were extracted from patient records. Ethical clearance was obtained.

Results: A total of 95 patients were started ART at the HIV clinic, Colombo during this period. Majority (74\%) were males with median age of 38 years. Among PLHIV $35.1 \%$ males and $24 \%$ of females were in WHO stage 3 or 4 at the time of presentation. TB was the commonest OI (10\%) followed by PCP (9\%). ART was started due to CD4 count< 500 cells/ $\mu \mathrm{l}(77 \%)$, key populations (6\%) and for PMTCT (5\%). ART regimen had to be substituted in $7 \%$. Adherence was satisfactory in females (88\%) and males (89\%). Most of the patients were on TDF+FTC+EFV regimen (67\%) and this group experienced least side effects. Quality of life improved in 95\%. CD4 count increased while viral load was $<1000$ copies $/ \mathrm{ml}$ among $96 \%$ of those who had satisfactory adherence $(n=76)$.

Conclusion: Most patients had satisfactory adherence and clinical, immunological and virological response was satisfactory. Poor adherence need to be further analyzed. Fixed dose combination TDF+FTC+EFV was the preferred first line regimen and was well tolerated by PLHIV.

Key words: ART, PLHIV, viral load, response, fixed dose combination

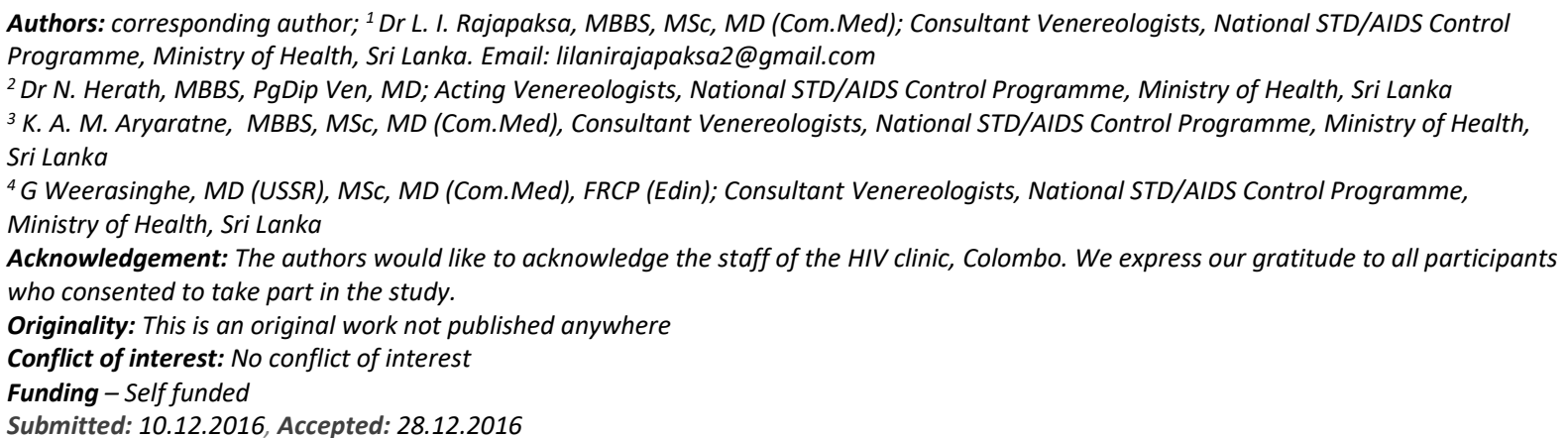




\section{Full article}

\section{Introduction}

By the end of 2015, 2309 new HIV infections have been reported to National STD/AIDS Control Programme with a HIV seropositivity rate of $0.02 \%$. (1) According to the data available, adult prevalence remains less than $0.1 \%$ and the estimated number infected in the community is 4200 . (2)

PLHIV are managed according to the national ART guidelines including appropriate treatment, contact tracing and regular follow up. Services for PLHIV are offered ranging from counselling and basic care to provision of ART services. (3)

The decision on starting ART depends on the stage of the disease, CD4 levels, viral load, presence of any concomitant opportunistic infections, pregnant women and key populations. The goal of treatment is to maintain satisfactory immune system to prevent the occurrence of opportunistic infections. In Sri Lanka the CD4 level considered for eligibility for ART was <200 cells/ $\mu \mathrm{l}$ from 2004. In the year 2011 this was changed to $<350$ cells $/ \mu$. The 2013 WHO consolidated guideline gives the cut off point as $<500$ cells $/ \mu$ l with priority for $<350$ cells/ $\mu$ l.(3) Antiretroviral therapy (ART) is the principal method for preventing decline of immunity. According to the 2013 WHO guideline prophylaxis for specific opportunistic infections was indicated when CD4 count is $<200$ cells/ $\mu$ l. (4)

Successful long-term ART results in a gradual recovery of CD4 cell numbers and an improvement of immune response. Viral load is expected to decline within 5-6 months to undetectable levels indicating the response to ART. In addition to virologic response and reduced risk of opportunistic infection, there is evidence to suggest that non-AIDS-defining illnesses, particularly in psychiatric and renal disease, may also be reduced when on ART. (4)
Treatment guidelines for HIV infection are age-specific. Guidelines for adults and adolescents are compiled by the National STD AIDS Control Programme (NSACP) and latest edition is in 2014.

\section{Justification}

Sri Lanka has initiated ART programme in 2004 and since then has managed many PLHIV with satisfactory results. The first guideline on HIV care was printed in 1998 followed by the ART guideline in 2004. $(5,6)$ This was recently updated in 2014 based on the global consolidated guideline of WHO. It is important to have a clear understanding on the use of guideline in the management of PLHIV and also to assess the clinical and laboratory response to ART. For this we should look at the PLHIV population identified, eligibility criteria for initiation of ART, side effects, adherence issues and response to ART including weight gain, improvement of clinical presentation, CD4 levels and viral load levels.

Clinical response will be assessed according to the WHO staging, performance scale and presence of opportunistic infections. WHO definitions for clinical, immunological and virological failure will be considered.

Clinical failure in adults - New or recurrent clinical event (other than IRIS) indicating WHO stage 4 condition after 6 months of effective treatment

Immunological failure - CD4 counts falls to the baseline or below, persistent CD4 levels below 100 cells $/ \mathrm{mm} 3$ or CD4 count drop by $50 \%$ or more from peak value

Virological failure - Plasma viral load above 1000 copies/ml based on two consecutive viral load measurements after 3 months of treatment (4)

We designed a prospective study to achieve the following objectives: 


\section{Objectives}

\section{General objective}

To determine the response to ART of adult PLHIV attending HIV clinic, NSACP

\section{Specific objectives}

To determine the sociodemographic characteristics of PLHIV on ART attending HIV Clinic, Colombo.

To determine the eligibility criteria to start ART

To describe the response to ART based on clinical as well as laboratory data

To describe the effect of side effects and adherence issues in ART services.

To determine the occurrence of drug resistance and treatment failure

\section{Methods}

It was a clinic based descriptive study.

\section{Study setting}

Study was conducted in the HIV clinic, Colombo for two-year period from 1st November 2014 till 31st October 2016. HIV clinic, Colombo is the main HIV clinic of the country which had been in existence since HIV care services started in Sri Lanka. The principal investigator and other investigators are permanent staff members having experience in the setting for more than three years.

\section{Sample size}

Study population was adult PLHIV eligible for ART services at the HIV clinic, Colombo. All PLHIV who become eligible for ART from 1st November 2014 to 31st October 2015 during were included in the sample. (5) According to the ART guideline developed in 2014 for Sri Lanka the eligibility criteria to start ART in 2014 and 2015 included the following;
CD4 count $<500$ cells $/ \mu$ l

WHO stage 3 or 4

Pregnancy

Key population

Sero-discordant couples

\section{Exclusion criteria}

The following categories were excluded from the sample: paediatric patients with HIV, pregnant women with HIV and those who cannot give information regarding partners such as mentally subnormal people. PLHIV who had got registered during this period but had previous exposure to ART also were excluded.

\section{Study instrument}

Data were collected by using an interviewer administered questionnaire which include questions on socio demographic characteristics, details about side effects and adherence issues. Emphasis was placed on clinical improvements following initiation of ART. Diagnosis of past opportunistic infections were based on the diagnosis cards issued during inward management and current infections were evaluated by a consultant physician. Details on the diagnosis of mild to moderate conditions which were managed as outpatients, eligibility criteria for ART and laboratory data were obtained through case records maintained at the HIV clinic.

\section{Method of data collection}

Data were collected by investigators who are practicing medical officers at the HIV clinic. As experienced medical officers having more than one year experience at the HIV clinic the investigators were able to obtain necessary information using correct techniques. They were given a 2-day training before conducting the study. 


\section{Collection of data}

Interview method was used to obtain information as this method also offers opportunity of counselling PLHIV as well. (7) There were 95 PLHIV who became eligible according to the criteria considered in the study.

Each eligible PLHIV started on ART was included in the study sample. They were informed in detail the purpose of the study. A friendly atmosphere was developed before starting the interview. These people were interviewed while taking the history or when they were waiting for laboratory results. Only one person was interviewed in a clinic room at a time. After getting the informed consent questions were introduced. All the responses were entered by the interviewers according to the previously defined way. After the completion of the questionnaire the respondents were counseled on ART services.

The sample was followed up till end October 2016 thereby end of the period all in the sample had completed period of one year or more after starting ART. Medical officers went through case records and previous diagnosis cards of each patient and filled the check list. Some cases which had issues in diagnosis were discussed with the consultant physician and final diagnosis was reached.

Diagnosis of past opportunistic infections were extracted from diagnosis cards issued during inward management and current infections were evaluated by a consultant physician. Details on the diagnosis of mild to moderate conditions which were managed as outpatients, eligibility criteria for ART and laboratory data were obtained through case records maintained at the HIV clinic.

\section{Method of data analysis}

Data was analysed by the principal investigator using SPSS package.

\section{Permission and ethical clearance:}

Ethical clearance was obtained from the Ethical clearance committee of the National Hospital Sri Lanka. Permission to conduct the study was obtained from the Director, National STD AIDS Control Programme, Sri Lanka. Interviewer administered questionnaire did not include personal identification details to maintain anonymity and confidentiality. Further medical officers who have satisfactory training at the STD clinic and having more than one year experience in providing services were used as interviewers.

\section{Results}

Among the PLHIV who started ART in the period $75.8 \%$ were males and median age of PLHIV was 38 years. Median age for males was 38.9 years and for females this was 38.6 years. Most of the PLHIV had education up to OL or above and more females (72\%) were in this group than males (68.4\%). More males (38.0\%) were single than females $(28.5 \%)$. Close to half of PLHIV (54.3\%) were married and living together with marital partner. Among males $53.5 \%$ were married while among females it was $57.1 \%$. Monthly family income was less than Rs. 20,000 for most of the PLHIV (77.0\%). More females belonged to this category (91.6\%) than males (71.4\%).

Among PLHIV 29\% was identified in the late stage with symptoms including two PLHIV referred following diagnosis of TB. Five females were diagnosed through ANC screening. More PLHIV were in stage 1 and 2 at the time of diagnosis (67.4\%) while $35.7 \%$ males and $22.7 \%$ females were in WHO stage 3 or 4 . There were more males in stage 3 and 4 than females. However, this difference was not significant. $(P>0.05)$ At the time of initiating ART significantly higher percentage of males $(31.4 \%)$ were ill in performance B or C stage than females (18.2\%).

Among PLHIV 69.6\% did not have any OI during the previous year and there was no 
difference between males (70\%) and females (68\%). The common opportunistic infections were TB (10.1\%), PCP pneumonia (9.1\%) and oral candidiasis (5.1\%).

Table 1: Eligibility criteria for initiation of ART

\begin{tabular}{lrrrrrr}
\hline Eligibility criteria & \multicolumn{3}{c}{ Male } & \multicolumn{2}{c}{ Female } & \multicolumn{2}{r}{ Total } \\
\cline { 2 - 7 } for ART & No. & $\%$ & No. & $\%$ & No. & $\%$ \\
\hline WHO stage & 2 & 2.9 & 2 & 9.1 & 4 & 4.3 \\
\hline CD4 count & 57 & 81.4 & 15 & 68.2 & 72 & 78.2 \\
\hline Sero-discordant & 4 & 5.7 & 1 & 4.5 & 5 & 5.4 \\
\hline Pregnancy & & 0.0 & 4 & 18.2 & 4 & 4.3 \\
\hline TB & 2 & 2.9 & 0 & 0.0 & 2 & 2.2 \\
\hline MARP & 5 & 7.1 & 0 & 0.0 & 5 & 5.4 \\
\hline Total & 70 & 100 & 22 & 100 & 92 & 100.0 \\
\hline (Missing-3) & & & & & &
\end{tabular}

Most patients were started on ART due to low CD4 count less than 500 cells/ $\mu$ l. Five males started ART as they were continuing risk behaviours as MARPs. Pregnant women and PLHIV in serodiscordant relationships were others who started ART. Three PLHIV who were diagnosed in late stage of infection died soon after diagnosis before starting ART.

The preferred first line regimen with fixed combination dose TDF+FTC+EFV was used for $66.3 \%$ of PLHIV. Fixed dose combination was the most used ARV regimen in all four WHO clinical stages. This included $70.8 \%$ males and $66.6 \%$ females. Another $11.6 \%$ of PLHIV were started on AZT+3TC+EFV. There were $12.6 \%$ on $\mathrm{PI}$ based regimens as first line ARV regimen. $\mathrm{PI}$ had to be considered in some instead of EFV due to neurological issues, depression, pregnancy and late stage presentations etc.

At the end of October 2016 there were 6 deaths reported in the sample and six were lost to follow up. Four PLHIV who died were in stage 4 at the time of starting ART.

In the sample, only $1 \%$ had highly unsatisfactory adherence. However, another 9.5\% showed unsatisfactory adherence. In relation to adherence there was no marked difference among males (11\%) and females (12.5\%). There was no association of adherence with educational status of PLHIV or marital status. However, those who had monthly income >Rs. 20,000 showed adherence more than those who earned less. Among those who were on TDF+FTC+EFV adherence was unsatisfactory in $12.6 \%$ while this was $20 \%$ among PLHIV on $\mathrm{TDF}+\mathrm{FTC}+\mathrm{LPV} / \mathrm{r}$.

\section{Side effects}

Of the sample, $68.4 \%$ did not experience any side effects due to ART. There was no difference in males (69.1\%) and females (65.0\%). The common side effects reported were vomiting and nausea (11.5\%) and skin rash (5.2\%). These were mostly seen in the first 3 weeks after starting ART. Significant side effects were few which included jaundice (1.1\%), anaemia (3.2\%) and drowsiness (1.1\%). $76.9 \%$ of PLHIV who were started on TDF+FTC+EFV did not show any side effects while this was $70 \%$ among PLHIV on $A Z T+3 T C+E F V$. Among those on TDF+FTC+LPV/r $54.5 \%$ were free of side effects. All 3 PLHIV who were on $A Z T+3 T C+L P V / r$ experienced significant side effects. As expected PLHIV who developed anaemia were on AZT based regimens. Drowsiness, headache and fatigue were experienced by PLHIV who were on EFV based regimens. Skin rash, nausea and vomiting was seen among PLHIV who were on TDF+FTC+EFV.

Only six PLHIV showed significant side effects to substitute first line ARV regimen. This included 2 patients on $A Z T+3 T C+L P V / r$ and 2 PLHIV on TDF+FTC+LPV/r. Those on fixed dose combination TDF+FTC+EFV only $2(3 \%)$ had to substitute $A R V$ regimen due to side effects.

When death and lost to follow up was excluded $94.5 \%$ showed improvement of quality of life while on ART. Five PLHIV including, four on TDF+FTC+EFV (6.1\%) and one on TDF+FTC+LPV/r (10\%) did not report 
improvement of quality of life following initiation of ART. This included one PLHIV who was in WHO stage 4 at the start of ART and four PLHIV who were in stage 1 and 2.

Mean weight increased from $53.3 \mathrm{~kg}$ at the time of initiating ART to $55.6 \mathrm{~kg}$ after starting ART. Median CD4 count at the time of starting ART was 276.7 cells $/ \mu$ land the range was 2 774 cells $/ \mu \mathrm{l}$. This increased to 530.8 cells $/ \mu \mathrm{l}$ after treatment. With range $23-932$ cells $/ \mu$ l. PLHIV in CD4 level less than 200 cells $/ \mu \mathrm{l}$ decreased markedly from $34.7 \%$ to $3.2 \%$ while PLHIV with more than 500 cells $/ \mu$ increased from $10.2 \%$ to $66.3 \%$ after starting ART.

By the end November 2016, there were six deaths reported in the group. Six PLHIV were lost to follow up while three were transferred out to other clinics.

By the end November 2016, out of 80 PLHIV who were tested for viral load $91.3 \%$ had viral load $<1000$ copies $/ \mathrm{ml}$ with $85 \%$ with viral load $<34$ copies $/ \mathrm{ml}$. Out of 7 who had $>1000$ viral load four had poor adherence. Three PLHIV showed treatment failure while on ART and they had low CD4 counts before starting ART. Among PLHIV who continued ART with satisfactory adherence $96.1 \%$ showed reduced viral load less than 1000 copies/ml.

Table 2: Change in viral load after ART for more than one year

\begin{tabular}{lrr}
\hline Viral load level & $\begin{array}{r}\text { No. of } \\
\text { PLHIV }\end{array}$ & Percent (\%) \\
\hline$<34$ & 68 & 85.0 \\
\hline $34-<1000$ & 5 & 6.3 \\
\hline$>1000$ & 7 & 8.7 \\
\hline Total & 80 & 100.0 \\
\hline
\end{tabular}

Satisfactory viral load reduction response was closely associated to the adherence level. Those who showed poor adherence had high viral loads at the end of one year period. There was no relationship between side effects experienced and the level of adherence.

\section{Discussion}

The main objective of the study was to determine the response to ART of adult PLHIV attending the main HIV clinic, Colombo. All adult PLHIV who started ART during the study period ( $n=95)$ were recruited for the study. Small sample size was a limitation. The instrument which was used to collect data was an interviewer administered pre-coded structured questionnaire given in the Annexures. Representing the country literacy level $98 \%$ had some form of schooling. However, it was noted that females had more education than males. Close to three fourths of the PLHIV had monthly income <Rs.20,000. The percentage of females receiving family income < Rs. 20,000 was higher than males.

More males were in late stage than females at the time of diagnosis. This may be due to low perception of risk. As seen elsewhere TB and PCP were the commonest OI among PLHIV. In addition to low CD4 counts and symptoms other causes such as serodiscordant relationship, pregnancy and key populations practicing risk behaviours too were considered as eligibility criteria for ART according to the guidelines. With ART close to 90\% showed improved quality of life. There were deaths among PLHIV diagnosed late in the disease with low CD4 counts. Early diagnosis would have prevented these deaths. According to the WHO recommendations most PLHIV were started on first line ART regimen with TDF+FTC+EFV. Side effects were not uncommon however, only six PLHIV had to substitute first line regimen due to toxicity. Serious toxicity which required substitution was lowest in the group on fixed dose combination TDF+FTC+EFV.

As expected with ART there was marked improvement of quality of life in $94.5 \%$ of PLHIV. After initiation of ART performance improved and mean weight increased. Median CD4 count increased from 276.7 cells $/ \mu$ lo 530.8 cells/ $\mu$ l. There was significant reduction in viral load with $91.3 \%$ of the sample showing viral load $<1000$ 
copies $/ \mathrm{ml}$. Weight gain and performance scale improvement too indicate the positive response to ART. Those who showed high viral loads were either having unsatisfactory adherence or were defaulters. One year later there were six deaths mainly among late presenters who had low CD4 counts at the start. Though adherence is considered as a major challenge, in this study a high level of adherence was observed in most patients.

A major limitation of the study was the small number of patients who participated. Being a low prevalence country annual case detection is between 200-225. Out of them the numbers becoming eligible for ART is further reduced.

The study can conclude that significant immunological improvement and virological suppression is possible if satisfactory adherence is observed. As seen in other countries fixed dose combination TDF+FTC+EFV was the preferred first line regimen and was well tolerated by PLHIV in the present study.

\section{References}

1. National STD/AIDS Control Programme. Ministry of Health, Annual Report 2015. Colombo; 2016

2. National STD/AIDS Control Programme. Ministry of Health, Sri Lanka country estimates HIV; 2015

3. National STD/AIDS Control Programme. Ministry of Health, The guidelines on use of antiretroviral drugs. Colombo: NSACP. (2014).

4. WHO. Consolidated guidelines on the use of ART. Geneva: WHO. 2013.

5. National STD/AIDS Control Programme. Ministry of Health, Guidelines for use of ART. Colombo: NSACP. 2004

6. National STD/AIDS Control Programme. Ministry of Health, Management of HIV infection. Colombo: NSACP. 1998

7. Fisher Andrew James R Foreit. Designing HIV AIDS intervention studies. Population Council. 8. WHO.The use of antiretroviral drugs for treating and preventing HIV infection WHO Geneva, second edition. 2016 